\title{
Electrophysiological and clinical assessment of dysautonomia in multiple system atrophy (MSA) and progressive supranuclear palsy (PSP): a comparative study
}

\author{
Monika Nojszewska, Anna Potulska-Chromik, Zygmunt Jamrozik, \\ Piotr Janik, Beata Zakrzewska-Pniewska \\ Department of Neurology, Medical University of Warsaw, Poland
}

\begin{abstract}
Clinical rationale for the study. Autonomic nervous system (ANS) involvement in different parkinsonian syndromes has been frequently discussed. It is well established in multiple system atrophy (MSA), whereas it is less evident in progressive supranuclear palsy (PSP).

Aims of the study. The aims were to assess the presence and pattern of ANS involvement in MSA and PSP using noninvasive tests i.e. the sympathetic skin response (SSR) test and the R-R interval variation (RRIV) test; to analyse the relationship between clinical and electrophysiological abnormalities in both disorders; and to assess whether an autonomic profile might help to differentiate them.

Materials and methods. Clinical and electrophysiological assessments of dysautonomia were performed in 59 patients with MSA ( 24 cases of MSA-C and 35 cases of MSA-P), these 59 cases including 31 females, mean disease duration $4.2 \pm 2.7$ years, mean age $60.3 \pm 8.4$ years, and in 37 patients with PSP (12 females, mean disease duration $4.6 \pm 3.6$ years, mean age $67.5 \pm 6.1$ years) and the results were compared to the results obtained from 23 healthy controls matched for age and sex.

Results. Clinical dysautonomia assessed by an Autonomic Symptoms Questionnaire was observed in $97 \%$ of the MSA patients and in $84 \%$ of the PSP patients. SSR was abnormal in $64 \%$ and RRIV was abnormal in $73 \%$ of MSA cases. In PSP cases, these figures were $78 \%$ and $81 \%$ respectively. Dysautonomia was clinically more pronounced in MSA compared to PSP $(p<0.05)$, whereas electrophysiological testing revealed frequently subclinical ANS damage in PSP patients.

Conclusions and clinical implications. Our results point to the complementary role of electrophysiological tests in the diagnostic work-up of dysautonomia in parkinsonian syndromes.

Key words: MSA, PSP, autonomic nervous system, dysautonomia, SSR, RRIV

(Neurol Neurochir Pol 2019; 53 (1): 26-33)
\end{abstract}

\section{Introduction}

Although generally believed to be uncommon, in fact autonomic disorders are ubiquitous in neurological disease, including movement disorders [1].

Sympathetic skin response (SSR) is a relatively simple electrophysiological test used in clinical practice to assess the reflex activity of sympathetic sudomotor pathways [2-5], and is employed to evaluate pre- and postganglionic sympathetic activity [2,3]. SSR has been used to assess ANS function in various peripheral and central neurological disorders $[2,4,6]$. On the other hand, R-R interval variability (RRIV) reflects the state of parasympathetic innervation of the heart [6], and gives an insight into sympathovagal tone [7]. Cyclic

Address for correspondence: Anna Potulska-Chromik, Department of Neurology, Medical University of Warsaw, Poland, e-mail: apotulska@wum.edu.pl 
deep breathing is the best validated stimulus; both afferent and efferent pathways are vagally mediated and inhibited by anticholinergic agents [1].

MSA is a rare and fatal neurodegenerative disorder that is characterised by a variable combination of parkinsonism, cerebellar impairment, and autonomic dysfunction [8]. PSP is also a neurodegenerative disorder with early postural instability and falls, vertical supranuclear gaze palsy, akinetic-rigid predominant and typically symmetric parkinsonism with poor response to levodopa, pseudobulbar palsy, and frontal release signs [9-12]. Nowadays, PSP can be divided into PSP-parkinsonism (PSP-P), PSP-Richardson's syndrome (PSP-RS) and into several other clinical subtypes [13], while MSA can be classified as either MSA-cerebellar (MSA-C), or MSA-parkinsonism (MSA-P) [14]. Diagnostic criteria have been proposed for the clinical diagnosis of PSP and MSA $[10,14,15]$. However, pathological brain examination post mortem remains the gold standard for diagnostic classification. The mean survival rate in patients with PSP has been estimated to be $6-7$ years $[9,16]$; in patients with MSA it is 6-10 years [17-19]. According to recent research in MSA, severe dysautonomia and the early development of combined autonomic and motor features are unfavourable predictors of survival [20]. In PSP, early dysphagia, cognitive symptoms, PSP-RS phenotype and urinary incontinence have been found to be highly predictive of shorter survival in some studies $[10,11,16,20$, 21], whereas sleep disturbances and possible hallucinations have been suggested in another study [22].

It is well established that pronounced autonomic failure appears early in MSA, whereas it is less evident in PSP [23-26]. The objectives of our study were: to evaluate the presence and pattern of ANS involvement in MSA and PSP using two noninvasive electrophysiological tests (SSR and RRIV); to analyse the relationship between clinical and electrophysiological abnormalities in both disorders; and to assess whether an autonomic profile might help to differentiate them.

\section{Materials and methods}

\section{Patients}

Clinical and electrophysiological assessments of dysautonomia were performed in 59 patients with MSA: 24 with a diagnosis of MSA-C [15 men (62.5\%)] and 35 with a diagnosis of MSA-P [22 women (62.9\%)], and in 37 patients with PSP (with classical Richardson's syndrome phenotype). The MSA and PSP diagnosis was probable in $48(81.4 \%)$ and 31 patients $(83.8 \%)$ respectively. In the rest of the patients, the diagnosis was possible. The mean age in the MSA group was $60.3 \pm 8.4$ years (range $40-79$ ), and in the PSP group it was $67.5 \pm 6.1$ years (range $58-80$ ). The mean disease duration was $4.2 \pm 2.7$ years (range 1-14) in the MSA group, and $4.6 \pm 3.6$ years (range 1-20) in the PSP group. In the MSA group, 31 patients $(52.5 \%)$ were treated with L-dopa (mean dose $861 \pm 391 \mathrm{mg}$ daily; range $200-1,800 \mathrm{mg}$ ) while in the PSP group 28 patients $(75.7 \%)$ received this treatment with a mean dose of $804 \pm 304 \mathrm{mg}$ (range: $300-1,600$ ). The mean age in the MSA-C group was $59.0 \pm 7.8$ years (range $49-79$ ), and in the MSA-P group it was $61.3 \pm 8.8$ years (range $40-78$ ). Patients with the presence of focal cerebral lesions in CT or MRI scans and other neurological or previously diagnosed severe systemic disorders (such as arterial hypertension or diabetes mellitus) or who were taking anticholinergic drugs, neuroleptics or drugs known to markedly influence autonomic functions (high doses of beta-blockers etc.), or with a history of alcohol or drug abuse were excluded from the study [27]. The control group consisted of 23 volunteers [ 16 women (69.6\%)] with a mean age of $56.6 \pm 14.0$ years (range $42-91$ ).

All patients were diagnosed and treated at the Department of Neurology, Medical University of Warsaw. The diagnosis of MSA was made according to the criteria established by Gilman et al. [14] and PSP according to the National Institute for Neurological Diseases and Stroke and The Society for PSP (NINDS-SPSP) [10] by movement disorders specialists (APCh, PJ, ZJ) based on a detailed history and a neurological examination. Antiparkinsonian treatment (mostly L-dopa medication) was not interrupted before examination, but the last dose was taken at least 24 hours before the examination took place.

\section{Methods}

Electrophysiological studies and clinical evaluations were performed at the Evoked Potential and Autonomic System Laboratory of the Department of Neurology, Medical University of Warsaw, between 2008 and 2015. All patients and controls gave informed consent to the protocol (for electrophysiological tests and clinical evaluation). The study protocol was reviewed and approved by the Bioethical Committee at the Medical University of Warsaw (No AKBE 13/2006). All the procedures were in accord with the standards of the Committee on Human Experimentation of the Medical University of Warsaw, and with the Helsinki Declaration of 1975.

\section{Clinical evaluation}

The clinical evaluation of dysautonomia was performed by two independent physicians (MN and BZP) on the same day as RRIV and SSR tests. We assessed the incidence and distribution of symptoms of dysautonomia as well as their intensity. We modified the Autonomic Symptoms Questionnaire proposed by Low [28] to evaluate the intensity of dysautonomia semiquantitatively using an arbitrarily defined score system (0 points - no symptoms; 1 point - symptoms present. Orthostatic hypotension was defined as a blood pressure decrease of $30 \mathrm{mmHg}$ systolic or $15 \mathrm{mmHg}$ diastolic within three minutes after standing up from a recumbent position according to MSA criteria [8]: 0 points - no symptoms; 1 point - mild $\rightarrow$ symptoms present only when there were 
facilitating conditions; 2 points - severe $\rightarrow$ symptoms present at all times, disabling).

\section{Electrophysiological tests}

SSR and RRIV tests were recorded in subjects lying in a semi-darkened room, with a temperature of $22-26^{\circ} \mathrm{C}$, after having relaxed for several minutes. Tests were recorded at the same time of day (between 10.00 and 13.00), within a few hours after a light meal using the Viking IV, Nicolet Biomedical Inc. (Multi-Mode Program Plus, version 4.0). Both tests were performed according to a protocol recommended by IFCN and described earlier [27]. The frequency of breaths during deep breathing was six per minute. The normal values were not corrected for age.

\section{SSR}

The latency and amplitude (peak to peak) of the highest response were measured (five evoked responses were registered, but only the one of the shortest latency was analysed). The SSR was considered abnormal if the latency was longer by more than two standard deviations (SDs) than that of the control group, or if a response was absent (i.e. not elicited by three consecutive stimulations). Additionally, we evaluated the degree of SSR abnormality using a five-level scale created in our Laboratory: 0 points - normal response; 1 point increased latency in one limb; 2 points - increased latency in both limbs or the absence of a response from one limb; 3 points - increased latency in one limb and the absence of a response from the other limb; 4 points - absence of response from both limbs.

\section{RRIV}

The RRIV result was considered abnormal if we registered decreased RRIV at rest or during deep breathing, or if no increase of the RRIV during deep breathing could be observed. Additionally, we evaluated the degree of RRIV abnormality using a three-level scale created in our Laboratory: 0 points normal RRIV test at rest AND during deep breathing AND an increase of the RRIV during deep breathing; 1 point - abnormal (decreased) RRIV test at rest OR during deep breathing OR no increase of the RRIV during deep breathing; 2 points - abnormal (decreased) RRIV test at rest AND during deep breathing AND no increase of the RRIV during deep breathing.

\section{Combined electrophysiological score}

A combined electrophysiological score, with values ranging from 0 to 6 , was created by combining the results from our SSR and RRIV scores.

\section{Statistical analysis}

Prior to analysis, the normality of distribution of the functional variables was tested by Shapiro-Wilk test. Where non-normal distribution was found, correlations analysis between different parameters was performed using Spearman's correlation coefficients test. For group comparison, Wilcoxon rank-sum, Chi-square and Fisher exact tests were used. Statistical significance was defined as $p<0.05$. Values are presented as mean $\pm \mathrm{SD}$. As an additional analysis, logistic regression was used to calculate the significance level because of the presence of confounding factors such as age and gender (in some group comparisons).

\section{Results}

The demographic profile of the two studied groups differed significantly: patients in the PSP group were older than patients in the MSA group ( $<<0.001)$, and the majority of them were male $(67.6 \%$ in the PSP group $v s 47.5 \%$ in the MSA group; NS). This difference was more clearly seen when the PSP group was compared to the MSA-P group which contained only 13 men $(31.1 \%, \mathrm{p}<0.05)$. The control group was matched for age and sex to the MSA group, but there were statistically significant differences between the control group and the PSP group. Volunteers were younger $(\mathrm{p}=0.001)$, and the majority of them were female $(\mathrm{p}=0.005)$.

\section{Clinical evaluation}

Clinical symptoms of dysautonomia were found in $96.6 \%$ (57/59) of our MSA patients and in 83.8\% (31/37) of PSP patients. The distribution of symptoms differed significantly between these groups, especially when orthostatic hypotension, dizziness and urinary incontinence were evaluated (Tab. 1).

When the MSA group was divided into MSA-P and MSA-C subgroups, orthostatic hypotension, urinary incontinence and constipation were significantly more often reported in the former. We did not find significant differences between MSA-C and PSP, although comparing PSP to MSA-P we found statistically significant differences in the frequency of all symptoms, except for urinary retention and constipation.

In semiquantitative evaluation of the intensity of dysautonomia, the mean score was higher in the MSA group as a whole (3.4 \pm 1.8 points) than in the PSP group (2.7 \pm 1.8 points), but this tendency did not reach statistical significance $(\mathrm{p}=0.054)$. When compared to PSP only, the MSA-P subgroup (mean $3.9 \pm 1.7$ points) results differed significantly. The intensity of clinical symptoms of dysautonomia was also more severe in the MSA-P subgroup than in the MSA-C (mean $2.7 \pm 1.7$ points) subgroup $(\mathrm{p}<0.05)$.

\section{Electrophysiological tests SSR}

The mean values of SSR from upper and lower limbs in controls, MSA and PSP patients are presented in Table 2. Technical reasons meant that we could not evaluate SSR results in three patients from each patient group.

The mean values of SSR latency in MSA and PSP patients were significantly higher than in controls for both the upper and lower limbs. 
Table 1. Frequency of symptoms of dysautonomia in MSA $(n=59)$ and PSP $(n=37)$ groups in Autonomic Symptoms Questionnaire for semiquantitative evaluation of dysautonomia. Modified from Low, 1997

\begin{tabular}{|c|c|c|c|}
\hline Symptom of dysautonomia & $\begin{array}{c}\text { MSA group } \\
\text { No of patients (\%) }\end{array}$ & $\begin{array}{c}\text { PSP group } \\
\text { No of patients (\%) }\end{array}$ & P value* \\
\hline Orthostatic hypotension & $41(69.5)$ & $19(51.4)$ & $P<0.05$ \\
\hline mild & $16(39)$ & $12(63.2)$ & \\
\hline severe & $25(61)$ & $7(36.8)$ & \\
\hline Urinary incontinence & $40(67.8)$ & $17(45.9)$ & $P<0.05$ \\
\hline Urinary retention & $18(30.5)$ & $15(40.5)$ & NS \\
\hline Impotence (in men) & $15(53.6)$ & $12(48)$ & NS \\
\hline Dizziness & $22(37.3)$ & $5(13.5)$ & $P<0.05$ \\
\hline Syncope & $20(33.9)$ & $8(21.6)$ & NS \\
\hline Constipation & $19(32.2)$ & $15(40.5)$ & NS \\
\hline
\end{tabular}

${ }^{*} \mathrm{P}<0.05$ Chi square test; NS - not statistically significant

Table 2. Latency of SSR test (in upper and lower limbs) and RRIV test results (at rest and during deep breathing) in MSA ( $n=59)$ and PSP $(n=37)$ patients and control group $(n=23)$

\begin{tabular}{|c|c|c|c|c|}
\hline & \multicolumn{2}{|c|}{$\begin{array}{l}\text { SSR latency (sec) } \\
\text { mean } \pm \text { SD (range) }\end{array}$} & \multicolumn{2}{|c|}{$\begin{array}{l}\text { RRIV test results (\%) } \\
\text { mean } \pm \text { SD (range) }\end{array}$} \\
\hline & upper limb & lower limb & $\mathbf{R}$ mean & R-DB \\
\hline \multirow[t]{2}{*}{ MSA } & $1.58 \pm 0.3^{*}$ & $2.11 \pm 0.3^{*}$ & $7.26 \pm 4.42^{* *}$ & $14.41 \pm 7.88^{* *}$ \\
\hline & $(1.04-2.42)$ & $(1.45-2.92)$ & $(2.31-27.0)$ & $(2.75-36.85)$ \\
\hline \multirow[t]{2}{*}{ PSP } & $1.63 \pm 0.24^{*}$ & $2.12 \pm 0.3^{*}$ & $5.78 \pm 2.23^{* *}$ & $12.19 \pm 6.84^{* *}$ \\
\hline & $(1.24-2.34)$ & $(1.68-2.97)$ & $(1.93-10.98)$ & $(2.26-29.40)$ \\
\hline \multirow[t]{2}{*}{ Control group } & $1.37 \pm 0.12$ & $1.89 \pm 0.23$ & $11.96 \pm 5.36$ & $29.86 \pm 16.53$ \\
\hline & $(1.12-1.60)$ & $(1.49-2.29)$ & $(4.96-24.07)$ & $(8.40-61.40)$ \\
\hline
\end{tabular}

MSA - multiple system atrophy; PSP — progressive supranuclear palsy; R mean — mean HRV at rest; R-DB - HRV during deep breathing; RRIV — R-R interval variation test; sec — seconds; SD — standard deviation; SSR - sympathetic skin response; * ${ }^{*}$ statistically significant changes compared to control group ( $p<0.05$, using $t$-Test); ${ }^{*}$ statistically significant changes compared to control group ( $p<0.05$, using Wilcoxon test)

In only 21 (35.6\%) patients with MSA and in eight (21.6\%) patients with PSP, the SSR results were within normal limits. In $11(18.6 \%)$ patients in the MSA group, a response was not registered in the upper limbs, and in 20 (33.9\%) patients a response was not registered in the lower limbs. These changes in the upper limbs were more pronounced in the MSA-P subgroup than in the MSA-C subgroup (22.9\% vs $12.5 \%$ respectively). In the PSP group, SSR was not registered in the upper limbs in $13(35.4 \%)$ patients and in the lower limbs in $21(56.8 \%)$ patients. The differences between the MSA and PSP groups were statistically significant (for both the upper and lower limbs).

The distribution of SSR abnormalities (in scores) in MSA and PSP patients is shown in Figure 1a.

\section{RRIV}

The mean values of RRIV response at rest and during deep breathing in controls, MSA and PSP patients are presented in Table 2. Technical reasons meant we could not evaluate RRIV results in five patients from each patient group.
All mean values of RRIV parameters in MSA and PSP patients were significantly lower than in controls.

Most of the patients reached the highest score of 2 points on our scale of RRIV changes intensity, with the highest rate in the MSA-P subgroup (51.4\%). Only in $16(27.1 \%)$ patients with MSA and in seven (18.9\%) patients with PSP were the results of RRIV normal (Fig. 1b).

\section{Combined electrophysiological score}

A combined electrophysiological score could be obtained in 51 MSA and 30 PSP patients. Only in eight (15.7\%) patients with MSA [three (15.0\%) with MSA-C and five (16.1\%) with MSA-P] and in two (6.7\%) patients with PSP were the results of both tests (SSR and RRIV) within normal limits. In the MSA patients, the intensity of change most frequently seen (29.4\%) was 'mild' (a score of 2). On the other hand, in the PSP group 'severe' changes (a score of 6 ) were found most often (30.0\%) but only in $9.8 \%$ of patients in the MSA group, although this tendency did not reach statistical significance $(\mathrm{p}=0.057)$ (Fig. 1c). Within the MSA group there was a tendency to 


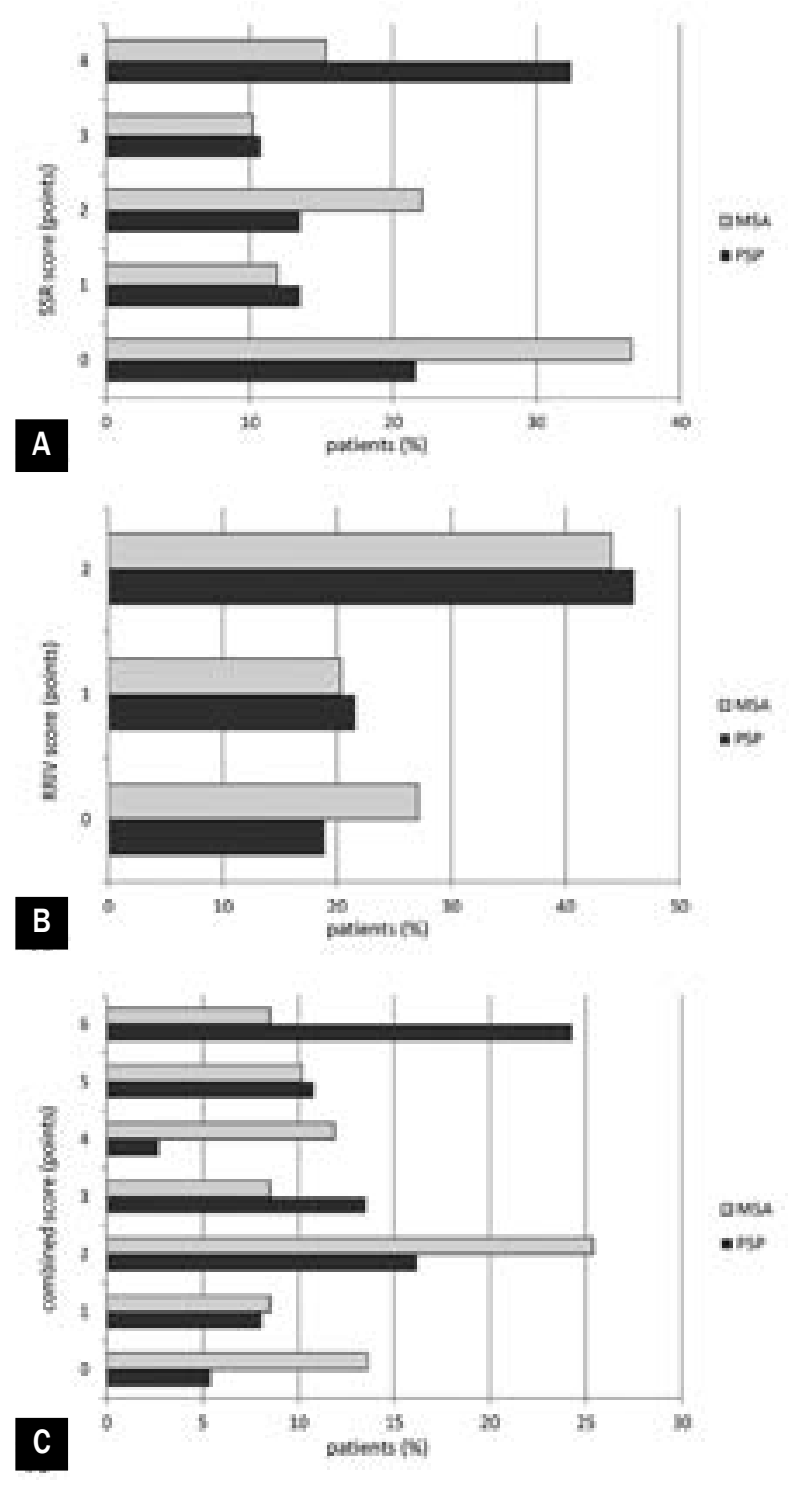

Figure 1. Distribution of intensity of SSR (1a) and RRIV (1b) abnormalities (in scores) and results in combined electrophysiological score $(1 c)$ in MSA $(n=59)$ and PSP patients $(n=37)$. MSA multiple system atrophy; PSP - progressive supranuclear palsy; RRIV - R-R interval variation test; sec - seconds; SD - standard deviation; SSR - sympathetic skin response

more severe changes (scores 5 and 6 ) in the MSA-P subgroup compared to MSA-C patients.

We found a mild, but significant, correlation between the results of SSR and RRIV tests in the PSP group $(r=0.38$; $\mathrm{p}<0.05)$. In the MSA group as a whole, as well as after division into subgroups, we found no such correlation.

\section{Correlation between clinical and electrophysio- logical assessments of dysautonomia}

The relationship between the presence and degree of electrophysiological changes (SSR and RRIV scores evaluated together and separately) and the intensity of clinical symptoms in the groups of patients with MSA and PSP were also analysed. In the MSA group, we found statistically significant correlations between clinical symptom score and SSR score as well as with the combined electrophysiological score (both $r=0.44 ; p<0.001)$. An even stronger correlation was found in the MSA-P subgroup $(r=0.57$ and $r=0.56$ respectively; $\mathrm{p}<0.001$ ). In PSP and MSA-C patients, no such correlation was found. No relationship between age, duration of disease and the values of the parameters analysed in the SSR and RRIV tests was revealed by a Spearman correlation test.

\section{Discussion}

Dysautonomia is common in parkinsonian syndromes affecting a wide spectrum of domains. It is well established that pronounced autonomic failure appears early in MSA, whereas it is less evident in PSP [23-26, 29].

In our study nearly $97 \%$ patients with MSA and $84 \%$ with PSP presented clinical signs of dysautonomia and they could be seen most frequently in the MSA-P subgroup. Our results are consistent with previous studies of MSA, where early and severe autonomic failure with predominant involvement of urinary and cardiovascular domains has been described as a key feature of the disease [19,30-33]. The ANS involvement in PSP is not so clearly established.

In our study the intensity of symptoms in PSP in most cases was mild. In some reviews, constipation and urinary incontinence have been described as the most prevalent non-motor features of PSP, especially in the late stages of the disease [22]. Other studies have noted orthostatic hypotension, dizziness, lack of sweating and sexual dysfunction $[16,21,23,24,34,35]$. Some authors have emphasised that comorbidities such as benign prostatic hypertrophy or medication use could explain some of these symptoms, because in many PSP patients objective evidence of autonomic dysfunction could not be found in diagnostic tests [22, 34, 36, 37]. There have also been reports that there are no prominent autonomic abnormalities in PSP [13, 25, 26, 29, 38], and the presence of cardiovascular symptoms has even been proposed as an exclusion criterion for PSP [25].

We found abnormal SSR results in 59\% of MSA patients and in $70 \%$ of patients with PSP. The pattern of involvement was distinct in both disorders, and those differences were statistically significant. The degree of SSR abnormalities was greater in the PSP than in the MSA group, and greater in the MSA-P than in the MSA-C subgroup.

Many studies have reported abnormalities in SSR results in $69 \%$, and even up to $100 \%$, of MSA patients [7, 35, 40-44]. Bordet et al. calculated the sensitivity of SSR examination in MSA diagnosis as 0.69 and the specificity as 0.92 [7]. On the other hand, Reimann et al. did not find differences in SSR results between MSA, PSP and PD groups [43], but they evoked SSR using acoustic stimulation. They also included in their study patients with significant comorbidities known to affect 
ANS function such as diabetes mellitus. The differences with our results could be caused by the SSR protocol applied. We used electrical stimulus to evoke SSR, because some of our patients with parkinsonism poorly cooperated during examination. This type of stimulus is easier to standardise in patients with central nervous system disorders $[7,39,40]$ and has been found to provide the most reproducible responses [5].

SSR involves both the pre- and postganglionic sympathetic systems $[2,3,6]$.The preganglionic efferent pathway is mainly the output of the intermediolateral column (ILC) of the spinal cord, which is frequently involved in the pathological process in MSA [19]. Such a central lesion might be the main determinant of abnormal SSR, as opposed to postganglionic dysfunction which can be seen for example in PD [7, 45].

Reports assessing SSR in patients with PSP and PD have shown that sudomotor function was markedly more involved in the former [46]. Pressor responses induced by emotional or physical stimuli or mental stress have also been reported to be diminished in PSP $[25,35]$. This type of sweating on the palms and soles is independent of the ambient temperature (so-called 'emotional sweating') and is regulated by the limbic system, motor system and reticular formation [35]. Many of these structures are known to be frequently affected by the neurodegenerative process in PSP $[7,12,22,48]$. Hence, similarly to MSA, sympathetic dysfunction in PSP may also be classified as of central, preganglionic origin.

There is also the possibility that the lack of SSR could be attributed to age. Drory and Korczyn reported that SSR could not been evoked in the hand in $27 \%$ of normal subjects above 60 years of age [39]. On the other hand however, Hay et al. reported the presence of responses in all elderly subjects [47]. Although there was a significant age difference between PSP patients and the control group in our study, a logistic model of analysis additionally proved that the differences in SSR results between both groups were independent of age.

We found abnormal RRIV results in $64 \%$ of patients with MSA and in nearly $68 \%$ of PSP patients, and in most cases the intensity of changes was severe.

Previous reports have described a lower mean value of heart rate variation (HRV) especially after deep breathing in over $60 \%$ of patients with MSA $[7,29,40]$. Pathological results have been found at all ages and within a short disease duration. However, other results did not confirm these findings [49].

Besides ILC, many ANS structures are affected by neurodegenerative changes in MSA, including dorsal vagal motor nucleus [19], which seems to be responsible for RRIV changes in MSA [7]. Because RRIV reflects the sympathovagal balance, so impairment in the sympathetic part of ANS reflected by SSR abnormalities might also contribute to RRIV changes [7].

Contrary to our results, previous reports have shown no remarkable changes in HRV in PSP [23, 25]. In the study conducted by Kikkawa et al. the mean HRV value at rest was lower in PSP than in the control group [35]. Holmberg et al. found only in four out of 14 patients with PSP decreased HRV during controlled deep breathing and limited hypotensive response during orthostatic provocation. In most cases (64\%), the results of both tests did not differ from controls [29].

Tau pathology in PSP is widely distributed in the brain, resulting in damage to various pathways [12,13]. It has been hypothesised that different PSP disease phenotypes might emerge from the preferential spread of tau through different brain networks that are functionally and neuroanatomically connected [13]. Therefore, we cannot exclude that in some patients neuropathological changes can spread and involve directly the parasympathetic structures responsible for RRIV changes in PSP. On the other hand, the involvement of sites important for the sympathovagal balance might also contribute to the RRIV changes seen in our patients with PSP.

Only in 16\% of MSA patients and in 7\% of PSP patients were the results of both electrophysiological tests within normal limits. The changes in laboratory tests were more pronounced in the PSP patients than in the MSA patients, but their distribution was not significantly different. We found a statistically significant correlation between the intensity of clinical symptoms of dysautonomia and the combined electrophysiological score in MSA patients, especially in the MSA-P subgroup. Other studies have found no such correlation in MSA [40].

\section{Clinical implications and future directions}

In parkinsonian syndromes, especially in MSA, a different degree of autonomic failure occurs that might significantly impair a patient's quality of life $[12,19,20,22,31,50]$. Therefore a systematic investigation of dysautonomia has been proposed as a relevant diagnostic assessment in parkinsonian syndromes [7, 33, 35, 40-42].

According to our results, sympathetic and parasympathetic involvement occurs in both MSA and PSP, but the intensity of changes varies between these two disorders and can be assessed by non-invasive electrophysiological tests. In MSA, a significant correlation between the intensity of clinical symptoms of dysautonomia and electrophysiological tests results can be found, whereas abnormal results of electrophysiological tests without clinical evidence of dysautonomia is more suggestive of PSP.

\section{References}

1. Weimer LH. Autonomic testing: common techniques and clinical applications. Neurologist. 2010; 16(4): 215-222, doi: 10.1097/ NRL.0b013e3181cf86ab, indexed in Pubmed: 20592565.

2. Fagius J, Wallin BG. Sympathetic reflex latencies and conduction velocities in normal man. J Neurol Sci. 1980; 47(3): 433-448, indexed in Pubmed: 7420119.

3. Fagius J, Wallin BG. Sympathetic reflex latencies and conduction velocities in patients with polyneuropathy. J Neurol Sci. 1980; 47(3): 449-461, indexed in Pubmed: 7420120. 
4. Shahani BT, Halperin JJ, Boulu P, et al. Sympathetic skin response--a method of assessing unmyelinated axon dysfunction in peripheral neuropathies. J Neurol Neurosurg Psychiatry. 1984; 47(5): 536-542, indexed in Pubmed: 6330307.

5. Elie B, Guiheneuc P. Sympathetic skin response: normal results in different experimental conditions. Electroencephalogr Clin Neurophysiol. 1990; 76(3): 258-267, indexed in Pubmed: 1697257.

6. Shahani BT, Day TJ, Cros D, et al. RR interval variation and the sympathetic skin response in the assessment of autonomic function in peripheral neuropathy. Arch Neurol. 1990; 47(6): 659-664, indexed in Pubmed: 2161208.

7. Bordet R, Benhadjali J, Destee A, et al. Sympathetic skin response and R-R interval variability in multiple system atrophy and idiopathic Parkinson's disease. Mov Disord. 1996; 11(3): 268-272, doi: 10.1002/mds.870110309, indexed in Pubmed: 8723143.

8. Gilman S, Wenning GK, Low PA, et al. Second consensus statement on the diagnosis of multiple system atrophy. Neurology. 2008; 71(9): 670-676, doi: 10.1212/01.wnl.0000324625.00404.15, indexed in Pubmed: 18725592.

9. Davis PH, Golbe LI, Duvoisin RC, et al. Prevalence and natural history of progressive supranuclear palsy. Neurology. 1988; 38(7): 10311034, indexed in Pubmed: 3386818.

10. Litvan I, Agid Y, Calne D, et al. Clinical research criteria for the diagnosis of progressive supranuclear palsy (Steele-Richardson-Olszewski syndrome): report of the NINDS-SPSP international workshop. Neurology. 1996; 47(1): 1-9, indexed in Pubmed: 8710059.

11. Santacruz P, Uttl B, Litvan I, et al. Progressive supranuclear palsy: a survey of the disease course. Neurology. 1998; 50(6): 1637-1647, indexed in Pubmed: 9633705.

12. Golbe LI. Progressive supranuclear palsy. Semin Neurol. 2014; 34(2): 151-159, doi: 10.1055/s-0034-1381736, indexed in Pubmed: 24963674.

13. Boxer AL, Yu JT, Golbe LI, et al. Advances in progressive supranuclear palsy: new diagnostic criteria, biomarkers, and therapeutic approaches. Lancet Neurol. 2017; 16(7): 552-563, doi: 10.1016/S14744422(17)30157-6, indexed in Pubmed: 28653647.

14. Gilman S, Low PA, Quinn N, et al. Consensus statement on the diagnosis of multiple system atrophy. J Neurol Sci. 1999; 163(1): 94-98, indexed in Pubmed: 10223419.

15. Payan CAM, Viallet F, Landwehrmeyer BG, et al. NNIPPS Study Group. Disease severity and progression in progressive supranuclear palsy and multiple system atrophy: validation of the NNIPPS--Parkinson Plus Scale. PLoS One. 2011; 6(8): e22293, doi: 10.1371/journal. pone.0022293, indexed in Pubmed: 21829612.

16. O'Sullivan SS, Massey LA, Williams DR, et al. Clinical outcomes of progressive supranuclear palsy and multiple system atrophy. Brain. 2008; 131(Pt 5): 1362-1372, doi: 10.1093/brain/awn065, indexed in Pubmed: 18385183.

17. Ben-Shlomo $\mathrm{Y}$, Wenning GK, Tison F, et al. Survival of patients with pathologically proven multiple system atrophy: a meta-analysis. Neurology. 1997; 48(2): 384-393, indexed in Pubmed: 9040727.

18. Wenning GK, Geser F, Krismer F, et al. European Multiple System Atrophy Study Group. The natural history of multiple system atrophy: a prospective European cohort study. Lancet Neurol. 2013; 12(3): 264-274, doi: 10.1016/S1474-4422(12)70327-7, indexed in Pubmed: 23391524.

19. Fanciulli A, Wenning GK. Multiple-system atrophy. N Engl J Med. 2015; 372(3): 249-263, doi: 10.1056/NEJMra1311488, indexed in Pubmed: 25587949.
20. Glasmacher SA, Leigh PN, Saha RA. Predictors of survival in progressive supranuclear palsy and multiple system atrophy: a systematic review and meta-analysis. J Neurol Neurosurg Psychiatry. 2017; 88(5): 402-411, doi: 10.1136/jnnp-2016-314956, indexed in Pubmed: 28250027.

21. dell'Aquila C, Zoccolella S, Cardinali V, et al. Predictors of survival in a series of clinically diagnosed progressive supranuclear palsy patients. Parkinsonism Relat Disord. 2013; 19(11): 980-985, doi: 10.1016/j. parkreldis.2013.06.014, indexed in Pubmed: 23968651.

22. Arena JE, Weigand SD, Whitwell JL, et al. Progressive supranuclear palsy: progression and survival. J Neurol. 2016; 263(2): 380-389, doi: 10.1007/s00415-015-7990-2, indexed in Pubmed: 26705121.

23. Gutrecht JA. Autonomic cardiovascular reflexes in progressive supranuclear palsy. J Auton Nerv Syst. 1992; 39(1): 29-35, indexed in Pubmed: 1629523.

24. van Dijk JG, Haan J, Koenderink M, et al. Autonomic nervous function in progressive supranuclear palsy. Arch Neurol. 1991; 48(10): 1083-1084, indexed in Pubmed: 1929903.

25. Kimber J, Mathias CJ, Lees AJ, et al. Physiological, pharmacological and neurohormonal assessment of autonomic function in progressive supranuclear palsy. Brain. 2000; 123 ( Pt 7): 1422-1430, indexed in Pubmed: 10869054.

26. Brefel-Courbon C, Thalamas C, Rascol O, et al. Lack of autonomic nervous dysfunction in progressive supranuclear palsy, a study of blood pressure variability. Clin Auton Res. 2000; 10(5): 309-312, indexed in Pubmed: 11198487.

27. Zakrzewska-Pniewska B, Gawel M, Szmidt-Salkowska E, et al. Clinical and functional assessment of dysautonomia and its correlation in Alzheimer's disease. Am J Alzheimers Dis Other Demen. 2012; 27(8): 592-599, doi: 10.1177/1533317512459792, indexed in Pubmed: 23007287.

28. Low PA. Clinical Autonomic Disorders: Classification and Clinical Evaluation. In: ed. Clinical Autonomic Disorders. Evaluation and Management. 2nd ed. Lippincott-Raven, New York. 1997: 3-13.

29. Holmberg B, Kallio M, Johnels B, et al. Cardiovascular reflex testing contributes to clinical evaluation and differential diagnosis of Parkinsonian syndromes. Mov Disord. 2001; 16(2): 217-225, indexed in Pubmed: 11295773.

30. Sakakibara R, Hattori T, Uchiyama T, et al. Urinary dysfunction and orthostatic hypotension in multiple system atrophy: which is the more common and earlier manifestation? J Neurol Neurosurg Psychiatry. 2000; 68(1): 65-69, indexed in Pubmed: 10601404.

31. Laurens B, Vergnet S, Lopez MC, et al. Multiple System Atrophy - State of the Art. Curr Neurol Neurosci Rep. 2017; 17(5): 41, doi: 10.1007/ s11910-017-0751-0, indexed in Pubmed: 28378233.

32. Iodice V, Lipp A, Ahlskog JE, et al. Autopsy confirmed multiple system atrophy cases: Mayo experience and role of autonomic function tests. J Neurol Neurosurg Psychiatry. 2012; 83(4): 453-459, doi: 10.1136/ jnnp-2011-301068, indexed in Pubmed: 22228725.

33. Kimpinski $\mathrm{K}$, lodice $\mathrm{V}$, Burton $\mathrm{DD}$, et al. The role of autonomic testing in the differentiation of Parkinson's disease from multiple system atrophy. J Neurol Sci. 2012; 317(1-2): 92-96, doi: 10.1016/j. jns.2012.02.023, indexed in Pubmed: 22421352.

34. Wenning GK, Scherfler C, Granata R, et al. Time course of symptomatic orthostatic hypotension and urinary incontinence in patients with postmortem confirmed parkinsonian syndromes: a clinicopathological study. J Neurol Neurosurg Psychiatry. 1999; 67(5): 620-623, indexed in Pubmed: 10519868. 
35. Kikkawa Y, Asahina M, Suzuki A, et al. Cutaneous sympathetic function and cardiovascular function in patients with progressive supranuclear palsy and Parkinson's disease. Parkinsonism Relat Disord. 2003; 10(2): 101-106, indexed in Pubmed: 14644000.

36. Sakakibara R, Hattori T, Tojo M, et al. Micturitional disturbance in progressive supranuclear palsy. J Auton Nerv Syst. 1993; 45(2): 101106, indexed in Pubmed: 8282945.

37. Valldeoriola F, Valls-Solé J, Tolosa ES, et al. Striated anal sphincter denervation in patients with progressive supranuclear palsy. Mov Disord. 1995; 10(5): 550-555, doi: 10.1002/mds.870100504, indexed in Pubmed: 8552104.

38. Asahina M, Vichayanrat E, Low DA, et al. Autonomic dysfunction in parkinsonian disorders: assessment and pathophysiology. J Neurol Neurosurg Psychiatry. 2013; 84(6): 674-680, doi: 10.1136/jnnp2012-303135, indexed in Pubmed: 22942216.

39. Drory VE, Korczyn AD. Sympathetic skin response: age effect. Neurology. 1993; 43(9): 1818-1820, indexed in Pubmed: 8414038.

40. De Marinis M, Stocchi F, Gregori B, et al. Sympathetic skin response and cardiovascular autonomic function tests in Parkinson's disease and multiple system atrophy with autonomic failure. Mov Disord. 2000; 15(6): 1215-1220, indexed in Pubmed: 11104208.

41. Asahina M, Kikkawa $Y$, Suzuki A, et al. Cutaneous sympathetic function in patients with multiple system atrophy. Clin Auton Res. 2003; 13(2): 91-95, doi: 10.1007/s10286-003-0072-z, indexed in Pubmed: 12720092.

42. Asahina M, Akaogi Y, Yamanaka Y, et al. Differences in skin sympathetic involvements between two chronic autonomic disorders: multiple system atrophy and pure autonomic failure. Parkinsonism Relat Disord. 2009; 15(5): 347-350, doi: 10.1016/j.parkreldis.2008.08.001, indexed in Pubmed: 18805037.
43. Reimann M, Schmidt C, Herting B, et al. Comprehensive autonomic assessment does not differentiate between Parkinson's disease, multiple system atrophy and progressive supranuclear palsy. J Neural Transm (Vienna). 2010; 117(1): 69-76, doi: 10.1007/s00702-0090313-y, indexed in Pubmed: 19763772.

44. Yokota T, Hayashi M, Tanabe H, et al. Sympathetic skin response in patients with cerebellar degeneration. Arch Neurol. 1993; 50(4): 422-427, indexed in Pubmed: 8460965.

45. Wang SJ, Fuh JL, Shan DE, et al. Sympathetic skin response and R-R interval variation in Parkinson's disease. Mov Disord. 1993; 8(2): 151-157, doi: 10.1002/mds.870080206, indexed in Pubmed: 8474481.

46. Sasaki I, Takeuchi H, Deguchi K, et al. [Autonomic nervous function in progressive supranuclear palsy-comparison with Parkinson's disease and healthy controls]. Rinsho Shinkeigaku. 1994; 34(10): 975-979, indexed in Pubmed: 7834957.

47. Hay JE, Taylor PK, Nukada H. Auditory and inspiratory gasp-evoked sympathetic skin response: age effects. J Neurol Sci. 1997; 148(1): 19-23, indexed in Pubmed: 9125386.

48. Homma S, Nakajima Y, Toma S, et al. Intracerebral source localization of mental process-related potentials elicited prior to mental sweating response in humans. Neurosci Lett. 1998; 247(1): 25-28, indexed in Pubmed: 9637401.

49. Gurevich TYu, Groozman GB, Giladi N, et al. R-R interval variation in Parkinson's disease and multiple system atrophy. Acta Neurol Scand. 2004; 109(4): 276-279, indexed in Pubmed: 15016010.

50. Brisinda D, Sorbo AR, Di Giacopo R, et al. Cardiovascular autonomic nervous system evaluation in Parkinson disease and multiple system atrophy. J Neurol Sci. 2014; 336(1-2): 197-202, doi: 10.1016/j. jns.2013.10.039, indexed in Pubmed: 24267739. 